\title{
Aggregated or disaggregated information first?
}

\begin{abstract}
Proliferation of reporting tools - such as "Online Analytical Processing" (OLAP) - and current practices of viewing information in a sequence from aggregated-to-disaggregated information are encouraged in the attention economy characterized by scarcity of human attention. The aggregated-to-disaggregated sequence in viewing information preserves scarce attentional resources by directing human attention. In a quasi-experimental setting, two sequences in viewing product profitability information - aggregated-to-disaggregated versus disaggregated-to-aggregated sequence - are contrasted. Results of 92 participating advanced accounting students reveal that consistent with conventional wisdom, the aggregated-todisaggregated sequence directs human attention. On the other hand, the disaggregated-toaggregated sequence facilitates knowledge application and discovery of previously undiscerned information that alters conventional understanding. Such findings shed light on the implications of directing human attention to preserve scarce attentional resources.
\end{abstract}

Keyword: Information aggregation; Performance monitoring; Knowledge; Human attention 